\title{
Comparative Study of Type-1 Fuzzy Logic and Type-2 Fuzzy Logic
}

\author{
Neeru Lalka \\ M.E., CSE \\ Thapar University, Patiala
}

\author{
Sushma Jain, PhD \\ Assistant Professor, CSED \\ Thapar University, Patiala
}

\begin{abstract}
Medical diagnosis is a complex process which can be attributed to the complexities, uncertainties and vagueness of the symptoms involved, and sometimes also because of their complex relationship with the final diagnosis output. Traditional systems for diagnosis very often incorporate certain inabilities that eventually lead to the vagueness in the result. Besides this, imprecise and incomplete knowledge are difficult for these traditional disease diagnosis expert systems to analyze. The fuzzy logic has carved a niche in medical diagnosis, for its ability to handle the dynamic nature of the disease diagnosis and medication. Various approaches of Fuzzy Logic, namely, Type-1 Fuzzy Logic, Interval Type-2 Fuzzy Logic, and General Type-2 Fuzzy Logic are being used for decision making in medical diagnosis. In this paper, a comparative study of the various parameters of Type-1 Fuzzy Logic and Interval type-2 Fuzzy Logic is conducted to understand their respective advantages in the medical diagnosis. Former, being a standard fuzzy logic methodology has been used widely for diagnosis of almost every disease, and the latter, which is also known as ' Layered Type-1 Fuzzy Logic', is being widely used for the diagnosis of a few diseases only. Type-1 Fuzzy Logic is rather a simple approach and results in the fast generation of outputs, but Type-2 Fuzzy Logic can provide better results in many cases. A study is conducted on type- 2 diabetes and heart related diseases, to understand the disease-specific nature of the two approaches. Type-2 Fuzzy Logic uses Karnik-Mendel (K-M) algorithm for type reduction. The comparison is drawn on the basis of accuracy, rule base and the differences of their outputs. In this way, this analysis helps to understand the advantages and disadvantages of both the approaches in the medical diagnosis.
\end{abstract}

\section{General Terms}

Type-1 Fuzzy Logic (T1FL), Interval Type-2 Fuzzy Logic (T2FL), Type-2 diabetes, Probability.

\section{Keywords}

Type-1 Fuzzy Logic (T1FL), Interval Type-2 Fuzzy Logic (T2FL), Centroid method, Rule Inference, Type-reduction, Rule aggregation, Apriori algorithm, Karnik-Mendel algorithm, Uncertainty indicator.

\section{INTRODUCTION}

Medical diagnosis and medication prediction, both are prone to various kinds of errors. Indirect and unknown relationships of the symptoms with the final output are very difficult to recognize and work with. Experts and doctors often diagnose the disease inaccurately when the symptoms overlap with that of some other disease. Besides this, the temporal nature of the symptoms may go unnoticed by the traditional diagnostic systems that basically operate on discrete information. The time-led variation in a disease changes its stage and demands up-to-date medication. In the era of telemedicine and ubiquitous self diagnostic systems, various anomalies in the prediction may go unnoticed in the absence of an expert or doctor.

Fuzzy logic has the tendency to handle the vagueness and uncertainty in a data set of any size. At the present scenario, importance of fuzzy logic based intelligent systems is recognized very well as it provides better accuracy and adapt to changes in the patterns of the disease diagnosis.

Lifestyle diseases are the long-term health conditions and require the medication to be administered over a long period of time. In such situations, it is imperative to accommodate the slightest change in the symptoms. The type-2 diabetes is attributed to the limited secretion of insulin and other factors like hereditary characteristics, diet, occupation, lack of workout, etc. are responsible for making diabetes condition worse. Some quantitative factors like glucose concentration in the body, body mass index, weight, etc. also affect the state of diabetes in an individual. Various intelligent approaches, namely, neural networks, fuzzy logic and genetic algorithm are being used to efficiently tackle the complexity of the attributes and for the optimizing rule base.

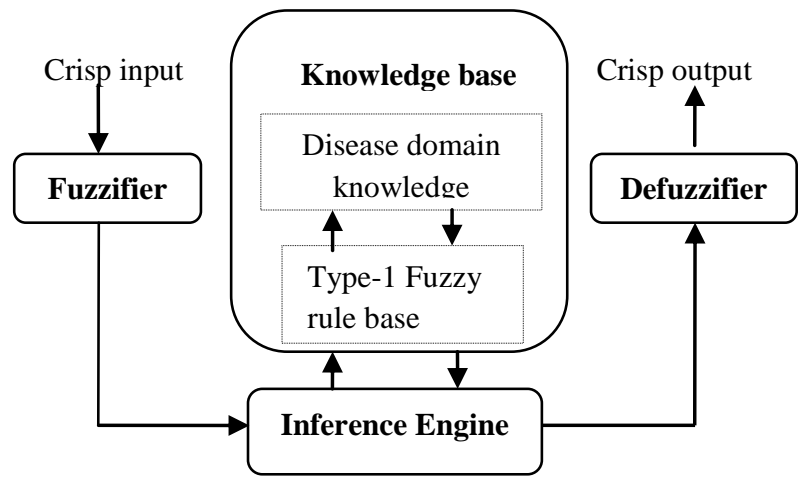

Fig 1: Type-1 Fuzzy Logic based expert system

In the heart diseases, the problem lies with the uncertainty of various risk factors, namely, resting blood pressure (trestbps), maximum heart rate achieved on arrival at the hospital (thalach), level of depression induced by exercise as compared to the value when at rest, i.e. oldpeak, etc. What encourages the use of fuzzy logic for diagnosis of heart related complications is the vagueness of distinction between a healthy individual and an unhealthy individual. T1FL has been extensively used for diagnosing the heart related problems, whereas there is a limited use of the Interval T2FL. The paper focuses on the use of Interval Type-2 FL for predicting heart diseases. Maintaining good 
accuracy becomes difficult when a diagnosis involves a large dataset and vague knowledge.

Type-1 Fuzzy Logic is the basic fuzzy logic methodology that performs fuzzification using the membership functions of various types depending on the requirement of an application. Fuzzy rule base basically stores the attributes as their fuzzy values where each fuzzy value incorporates a range of fuzzy values. The components of T1FL are shown in figure 1.

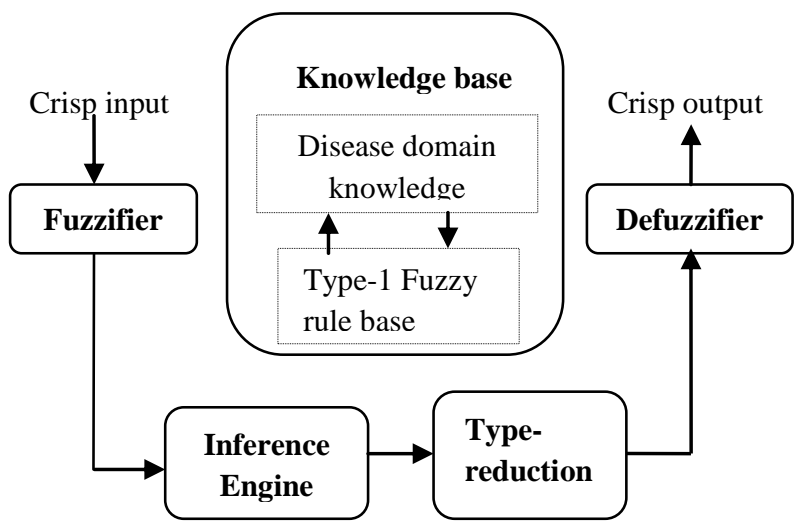

Fig 2: Type-2 Fuzzy Logic based expert system

Interval Type-2 FL is a layered architecture of Type-1 FL. In Interval Type- 2 FL, there is a fuzzifier that fuzzifies the crisp value into its type-2 fuzzy variant i.e. upper fuzzy value and lower fuzzy value, plus the corresponding type-1 fuzzy value. Rule inference is performed on these three fuzzy values for each consequence, i.e. 0 and 1 . Type-reduction is performed to change these type-2 fuzzy values into their corresponding type-1 fuzzy values. A brief description is shown in figure 2 . Section 2 discusses various fuzzy logic based medical diagnostic systems. Section 3 presents the proposed study and section 4 discusses the results. Section 5 will discuss the conclusion and future scope.

\section{LITERATURE REVIEW}

Lee and Wang [1] have proposed a diabetes fuzzy ontology for diagnosing diabetes. The ontology identifies the respective class membership of the patient and then an individualspecific diagnosis is performed. The knowledge base is created by using the attributes and the fuzzy relations formed from the fuzzy variables and the fuzzy numbers. Calegari and Sanchez [2] have proposed fuzzy based layered ontology architecture. Fuzzy ontology exploits the semantic correlation to define fuzzy relationships and the knowledge base efficiently. Abadi et al. [3] have proposed the use of recursive least squares for finding the relationship between inputs and the outputs in the diabetes expert system. This kind of depiction of fuzzy relationships and the corresponding model can be evolved according to the requirements. Also uncertainties in the weight, quantity of meals, time and lifestyle can be used while performing the diet recommendation. Gadoras and Mikhailov [4] have proposed a categorization mechanism for categorizing an input. It incorporates the generation of the rules; verifying them using the verification framework; class and knowledge representation using fuzzy sets, and identification of the new symptoms using the rules. The authors also proposed the use of fuzzy logic for handling uncertainty regarding the class memberships.
Yager and Petry [5] have proposed a methodology summarization of the data. Summarization helps in using the attribute hierarchies to control the data usage for performing the decision-making. Attribute hierarchies are constructed using the fuzzy sets. Summarization, in order to be accurate should have minimum coverage, maximum relevance, succinctness, and usefulness. The levels of summarization can be fuzzy in nature. Palma et al. [6] have proposed a methodology to evaluate a disease in time-specific manner. The procedure considers the time-specific features and the patterns of such dynamism regarding a disease. Then the parameters are modeled by Temporal Constraint Networks (TCN) which uses fuzzy logic. Seising [7] has proposed the use of fuzzy sets and fuzzy relations for the efficient representation of the vague classes.

Straszecka [8] has proposed a fuzzy based approach for handling the uncertainty in disease diagnosis and the vagueness of the symptoms. The model represents symptoms as focal points, and uncertainty, significance of a particular symptom in terms of a value from 0 to 1 , on the basis of their level of .significance. Fuzzy sets are used to represent these focal points. To measure the risk, the probabilities of the various risk factors are added.

Kalpana and Kumar [9] have proposed a fuzzy based determination procedure for improving the accuracy of diabetes diagnosis. Fuzzy determination mechanism (FDM) incorporates the fuzzy inference, fuzzy implication, and the fuzzy aggregation. Das and Kar [10] have proposed the use of fuzzy soft approaches for designing diagnosis algorithms. The algorithm involves analyzing the considerations of a group of experts who give their opinion only regarding the symptoms they have knowledge about. Their opinion is assigned confidence using the soft fuzzy approach, and later on, these confidence values are aggregated to make an accurate decision. Mahfouf et al. [11] have proposed the fuzzy logic as an optimal solution to model the complex biological problems, medical diagnosis being one of them. Experiments on the use of fuzzy logic in biological problems started in 1980s. Innocent and John [12] have proposed a fuzzy diagnostic system that incorporates the temporal information of a disease and in this way, fuzzy logic has been used to represent time-specific information regarding a disease. Symptoms are studied against a constraint-satisfaction system. According to which only if all the symptoms collectively satisfy the positive diagnosis at a particular time, the patient is a diagnosed as diabetic (in case of diabetes). This system can be developed using type-2 fuzzy logic.

Kahramanli and Allahverdi [13] have proposed an approach for diagnosis by collaborating the neural networks and the fuzzy logic. ANN reduces the complexity which is due to the multiple dimensions of the symptoms. Using neuro-fuzzy model, continuous data can be represented efficiently and helps in accurate classification. Gupta et al. [14] have proposed the use of neuro-fuzzy approach for the treatment of diabetes. ANN performs the fine tuning of the rules formulated by the fuzzy logic. This approach is used for learning and adaptation of diagnosis framework. Anouncia et al. [15] have proposed the use of fuzzy rough sets for the accurate representation of knowledge base.

Lee et al. [16] have proposed a type-2 fuzzy ontology in which an ontology model is mapped on Type 2 Fuzzy Sets (T2FS). The T2FS is useful for personal diet recommendation. The recommendation system incorporates the Type-2 Fuzzy Personal Profile Ontology (T2FPPO), Type-2 Fuzzy Food Ontology (T2FFO), and Type-2 Fuzzy Profile Food Ontology 
(T2FPFO). Mendel et al. [17] have proposed I-T2FL as more powerful than T1FL, in terms of dealing with fuzziness. Type2 fuzzy is more accurate as they model uncertainties in membership functions too. The author also discussed General Type-2 Fuzzy Logic (GT2FL) which can be formed by layers of I-T2FL. The Karnik-Mendel (K-M) type reduction algorithm is used for non-fuzzy problems too.

Yeh et al. [18] have proposed a modified type reduction algorithm in place of the K-M algorithm. The author highlighted the inefficiency of initialization of switch points in $\mathrm{K}-\mathrm{M}$ algorithms. The new algorithm, iteratively computes switch points and speeds up the process of convergence with previously calculated switch points. Liu's algorithm avoids regular iteration done by the K-M algorithm to compute switch points. Aladi [19] has proposed the efficient calculation of the FoU for the triangular membership function to provide a uniform range of uncertainty over the whole membership function. Using the Uncertainty Indicator, one can efficiently calculate the left and right switch points.

Delgado et al. [20] have proposed a closed loop approach for glucose-insulin control regime. Monitoring of insulin dosage is done on a daily basis and besides this a short duration dose control regime is followed before each meal in a day. Frequent measurement of glucose is done in order to achieve accuracy. A target of glucose level is set for each day, which can change according to variations in metabolism in an individual. Grant [21] has proposed using fuzzy as the artificial pancreas to regulate insulin dosage in a diabetic. The framework uses a feedback model with closed loop to regulate glucose in a diabetic with the help of biomedical sensors, instead of regular blood tests and other medical tests. The author used fuzzy to adjust the meager differences of the two glucose readings to administer insulin effectively.

Nazari et al. [22] have proposed a fuzzy model for controlling diabetes mellitus using a recursive least square method. The model involves controlling diet regime among the patients using inputs like weight, time of meals, duration of the simulation and glucose concentration in the fuzzy expert system. Body sugar and carbohydrate intake level is decided each day using MATLAB based glucoSim Simulator.

Adeli [23] have proposed the fuzzy based expert system for heart disease diagnosis. The author uses the Mamdami based inference method and compares the obtained results of the diagnosis with those in the database. The expert system designed here stimulates the way an expert and doctor relation to design a more accurate disease diagnosis system.

\section{IMPLEMENTATION}

\subsection{Type-1 Fuzzy Logic for diagnosis and medication prediction}

Type-2 diabetes disease prediction: As stated earlier, diabetes diagnosis is performed using various interrelated symptoms and attributes. The knowledge base constitutes the type-2 diabetes domain and the corresponding fuzzy values. The PIDD database from American Diabetes Association is used for diagnosis. Fuzzification is performed on the knowledge base using a trapezoidal membership function. Various attributes that are considered while diabetes diagnosis are:

- $\quad$ Three hour fasting glucose test (fgt)

- Body mass index (bmi)

- Diabetes min. blood pressure (dmbp)

- $\quad$ Skin fold thickness (tft)
- $\quad$ Serum insulin test (si)

- Diabetes mellitus function (df)

- Diabetes mellitus (D) - class variable

- Age

While medication prediction, two attributes namely, bmi and si are used and the class variable is 'td', i.e. total insulin dosage.

I. Type-2 diabetes knowledge base: The knowledge base has domain specific knowledge and the corresponding fuzzy values. Besides this, it also has a fuzzy rule base as shown in figure 1. Before fuzzification, maximum, minimum, mean and standard deviation is calculated for each attribute in the data set.

a) Construction of fuzzy numbers: For fuzzification, the fuzzy numbers are used. The three fuzzy numbers for each attribute are shown below, for 'si' attribute:

si_low:p=min(si), $q=\min (\operatorname{si}), \quad r=\operatorname{mean}(\operatorname{si})-\operatorname{stddev}(\operatorname{si}), \quad s=$ mean(si)

si_med:p=mean(si)-stddev(si), $\quad q=$ mean(si), $\quad r=$ mean(si), $p=$ mean $(a t t)+\operatorname{stddev}(a t t)$

si_high:p=mean(si), $\quad q=$ mean(si)+stddev(si), $\quad r=\max (s i)$, $s=\max ($ si $)$

b) Fuzzification: In T1FL, trapezoidal membership function is used. The membership function of each attribute is computed for all the fuzzy numbers. Using (1), three membership functions for each attribute for low, med and high fuzzy numbers $[\mathrm{p}, \mathrm{q}, \mathrm{r}, \mathrm{s}]$ are computed, where $\mathrm{p}$ is 'begin', $\mathrm{q}$ is 'after begin', $\mathrm{r}$ is 'before end' and $\mathrm{s}$ means 'end'.

$$
M=\left\{\begin{array}{c}
0, M<p \text { or } x>s \\
\left(\frac{M-p}{q-p}\right), p \leq x<q \\
1, q \leq x \leq r \\
\left(\frac{s-M}{s-r}\right), r<x \leq s
\end{array}\right.
$$

c) T1FL based rule base for diabetes: The rule base is formulated using the apriori algorithm of the WEKA data mining tool. The If-then-else rules are constructed using the five attributes, namely, fgt, sit, age, df and bmi. D which stands for diabetes mellitus, is the target variable. The rules are constructed using a fuzzy domain knowledge base. One such rule is given below.

$$
\begin{aligned}
& \text { if (fgt=='high') } \\
& \text { if (bmi=='med' \&\& df=='high') } \\
& \text { then } D=\text { ='tested positive'; } \\
& \text { else if (bmi=='med' \&\& df=='low' \&\& age=='low') } \\
& \text { then } D=\text { ='tested_negative'; }
\end{aligned}
$$

II. Diabetes diagnosis decision-making: The decision-making process starts when a valid input is received. The fuzzification is performed on input values. Then decision making further involves rule matching, rule inference and rule aggregation, and defuzzification for generating the output.

a) Type-1 fuzzification: The fuzzification of the input instance is performed as given in (1). The fuzzy values of all fuzzy numbers are stored in the dataset.

$$
\begin{gathered}
r_{a g g}=\operatorname{MAX}\left(r_{i n f}(i)\right) \\
\mathrm{Dm}=\sum_{i=1}^{v}(w(i) * y(i)) / \sum_{i=1}^{v} y(i)
\end{gathered}
$$


b) Rule inference: A set of 60 rules has been used, so matching degree will be calculated for 60 times ranging from $(0-1)$. The rule inference value is the minimum of the matching degrees of the rules with similar consequences. The result is a set of rules for each consequence, i.e., $\left[r_{i n f_{i}}, r_{i n f_{i+1}} r_{i n f_{i+2}} \ldots \ldots . . r_{i n f_{v}}\right]$, where $\mathrm{v}$ is the number of fired rules for each consequence and $r_{i n f_{i}}$ is the rule inference value. Then from these values, rule aggregation value, i.e. max of the inferred rule values as shown in (2) for each consequence is calculated.

c) Defuzzification: Then using (3), i.e. centroid method, where $w(j)$ is the weight of the aggregated rule value and $\mathrm{j}=[1,2]$, the final probability is computed.

d) Semantic description of the output: The probability of diabetes is divided into five parts, i.e. very low, low, medium, high and very high; if the crisp value of probability lies between 0 and 0.20 , the probability is very low, if between 0.20 to 0.40 , it is low, if between 0.40 to 0.60 , it is a medium, if between 0.60 to 0.80 , the probability is high and if it is between 0.80 to 1 or equal to 1 , it is very high.

The steps of computing probability for heart diseases are similar to that of diabetes. A heart related complications data set from the UCI machine learning repository is used for computing the disease prediction. Then T1FL fuzzification is performed on the dataset, rule base is formed, and both the fuzzy dataset and the rule base are stored in the knowledge base. The attributes that are used for the heart disease diagnosis are listed as follows:

- $\quad$ Chest pain type (cp)

- Exercise induced anigma (exang)

- $\quad$ ST depression induced by exercise (oldpeak)

- $\quad$ Slope of peak exercise (slope)

- Maximum heart rate achieved (thalach)

- Resting blood pressure (trestbps)

- $\quad$ Probability $(\mathrm{P})$

- Angiographic disease status (num)

Construction of fuzzy numbers for these attributes takes place as shown above for diabetes. The fuzzification is performed as shown in (1).

Heart disease diagnosis rule base: A rule base is constructed using the WEKA data mining tool. Apriori algorithm of the association tool is used to construct 50 rules. The target class is the 'num' attribute which can have any value from the set $[1,2,3,4]$. Some of the rules so formed are shown below:

1. if ( $\mathrm{cp}==$ 'high' \&\& age $=$ 'low' \&\& trestbps $=$ 'low' \& \& thalach $=$ 'med')

if (exang='low')

then num $=2$;

else if(exang='high')

then num $=3$;

2. if $(\mathrm{cp}==$ 'high' \&\& age='low'\&\& trestbps='low' \&\& thalach='low')

if (exang='low')

then num $=4$;

else if(exang='high')

then num $=2$;

After fuzzification, rule inference is performed using the fuzzy MIN operation for the four different consequences. Rule aggregation is performed as shown in (2). And defuzzification combines the results of different consequences using the centroid method.

Insulin dosage recommendation: Insulin dosage prediction uses the same methods under the T1FL as shown above for diabetes and heart diseases. Here, there are three consequences, i.e. low dosage, med dosage and high dosage for which rule inference is performed.

\subsection{Interval Type-2 Fuzzy Logic for diagnosis and medication prediction}

T2FL has three dimensional membership function and a type reducer to change the rule inference value to rule aggregation value that is based on T1FL. It works as a 'layered T1FL'.

I. Construction of knowledge base: The knowledge base consists of the domain knowledge of type-2 diabetes. As there are upper and lower membership function values corresponding to a type- 1 fuzzy value, the knowledge base is expanded. For an instance of the diabetes dataset, there will be around 15 fuzzy numbers.

II. Rule base construction: Rule base is constructed using apriori algorithm. Rule no. 6 and 8 of the T2FL based rule base for diabetes diagnosis are shown below.

6. if $(\mathrm{df}==$ 'low' \&\& age $==$ 'high') if ( $\mathrm{pgc}==$ 'high' \& \& bmi='med') then $\mathrm{D}=$ 'tested_positive';

$$
\begin{aligned}
& \text { 8. if }(\mathrm{df}==\text { 'low' \& \& age== 'high') } \\
& \text { if ( } \mathrm{pgc}==\text { 'med' \& \& bmi='high') } \\
& \text { then } \mathrm{D}=\text { ='tested_negative'; }
\end{aligned}
$$

III. T2FL based diabetes decision making: The steps are similar as type-1 fuzzy logic based decision making except the inclusion of type-reduction module before defuzzification. Now the crisp input is converted into its three dimensional fuzzy based variant

a) Construction of FoU: FoU means Footprint of Uncertainty which exists around the trapezoidal membership function for all the attributes and it can be uniform or nonuniform. In the proposed work, a uniform FoU around the membership function is used. It is calculated using the upper and lower membership functions, which are shown in (4) and (5)

$$
\begin{gathered}
\bar{\mu}_{\tilde{A}}=\min \left(\mu_{\tilde{A}}+\frac{c}{2}, 1.0\right) \\
\underline{\mu}_{\tilde{A}}=\min \left(\max \left(\mu_{\tilde{A}}-\frac{c}{2}, 0.0\right), 1.0-c\right) \\
F o U=\operatorname{MAX}\left(\bar{\mu}_{\tilde{A}}, \underline{\mu}_{\tilde{A}}\right)
\end{gathered}
$$

(6) Shows that FoU is the max of the two membership functions for a particular attribute. The value of the constant $\mathrm{C}$ which is used in the (4) and (5) should lie between 0 and 1 because the final probability lies between 0 and $1 . \mathrm{C}=0$ means negligible FoU and $\mathrm{C}=1$ means extremely wide FoU.

b) Calculation of Indicator of Uncertainty: Such an indicator helps to compute the level of uncertainty captured by the FoU for the attributes. It is usually the difference of the upper and lower membership function i.e. $\bar{\mu}_{\tilde{A}}-\underline{\mu}_{\tilde{A}}$.

c) Fuzzification: Trapezoidal membership function with FoU is used to find the fuzzy values of an instance as shown in (1). In this way, for each attribute there are three fuzzy 
values where the upper fuzzy value > T1FL fuzzy value $>$ lower fuzzy value.

$r_{\text {inf }_{\mathrm{i}}}=f u z z y(\text { age })_{i} * f u z z y(s i t)_{i} * f u z z y(b m i)_{i} *$

$f_{u z z y}(p g c)_{i} * f u z z y(d f)_{i}$

d) Rule inference for each consequence: The rule base is loaded in the fuzzy inference engine. The matching degree for each rule is calculated and only those rules are considered to be fired where the matching degree is greater than 0 . After that, rule inference is performed by using $\mathrm{t}$-form production method for each consequence, which means the product of the matching degrees as shown in (7). At the end of this step, there will be two sets of the fired rules, i.e. for 0 and 1 .

e) Type-reduction: The proposed work uses the Karnik and Mendel (K-M) type-reduction algorithm. The fired rules have inference values on which the type-reduction is performed. First step is the calculation of $\mathrm{T}$ for each consequence, i.e. switch points. $\underline{T}$ and $\bar{T}$ which are left and right switch points respectively.

$$
\begin{aligned}
& P_{j}=\left(\bar{R}_{j}+\underline{R}_{j}\right) / 2 \\
& \underline{T}=N / 2.4 \\
& \bar{T}=N / 1.7 \\
& A=\sum_{i=1}^{\underline{T}_{i}} x_{i} * \bar{I}_{i, j}+\sum_{i=\underline{T}_{i}+1}^{n} x_{i} * \underline{I}_{i, j} \\
& \bar{M}_{j}=A / \sum_{i=1}^{\underline{T}_{i}} \bar{I}_{i, j}+\sum_{i=\underline{T}_{i}+1}^{n} \underline{I}_{i, j} \\
& B=\sum_{i=1}^{\bar{T}_{i}} x_{i} * \underline{I}_{i, j}+\sum_{i=\underline{T}_{i}+1}^{n} x_{i} * \bar{I}_{i, j} \\
& \underline{M}_{j}=B / \sum_{i=1}^{\underline{T_{i}}} \underline{I}_{i, j}+\sum_{i=\underline{T}_{i}+1}^{n} \bar{I}_{i, j}
\end{aligned}
$$

Where, $\bar{M}_{j}$ is the left switch value,

$\underline{M}_{j}$ is the right switch value, where $\mathrm{j}$ is the type of the consequence

$x_{i}$ is the secondary membership function value or type-1 fuzzification base value,

$\bar{I}_{i, j}, \mathrm{I}$ is the rule inference value and $\bar{I}$ is the value of the rule inference in the upper FOU.

The initial values of the left switch points and the right switch point is given in (9) and (10), where $\mathrm{n}$ is the total number of fired rules. Then upper and lower type reduced fuzzy rule aggregation value is calculated as shown in (12) and (14). Before that $\mathrm{A}$ and $\mathrm{B}$ are calculated. Here, $\bar{T}$ and $T$ are the upper and lower type reduced values. ' $i$ ' points the particular rule on which type reduction is being performed. $j$ points to the consequence for which type reduced upper and lower values are being computed and here $j=[0,1] . x_{i}$ is the secondary membership function for each rule, i.e. type-1 fuzzy based rule inference value and $\bar{I}_{i, j}$ is the upper rule inference value, and $\underline{I}_{i, j}$ is the lower rule inference value. In this way, there are two type reduced sets for diabetes diagnosis. Using (8), the Type-2 FL based rule aggregation values are computed for the two consequences and are later defuzzified to get the probability. f) Defuzzification: Using the centroid method as stated in (10), the final probability is calculated. Here, $w(i)$ is the weight of aggregated fired rules for a consequence, $i=[0,1]$. And the $y(i)$ is the corresponding type-reduced type- 1 fuzzy value.

In the case of heart diseases, the procedure again remains the same besides the knowledge base which is specific to the domain of heart related diseases. Similarly, for the insulin dosage prediction the only change is the knowledge base

\section{RESULTS AND DISCUSSION 4.1 Evaluation Parameters}

I. Probability: The prediction of the disease diagnosis can be very low, low, med, high and very high, as shown in table 1 .

Table 1. Semantic description of the probability of the disease diagnosis

\begin{tabular}{|c|c|}
\hline Probability & Semantic description \\
\hline $0-0.20$ & very low \\
\hline $0.21-0.40$ & low \\
\hline $0.41-0.60$ & med \\
\hline $0.61-0.80$ & high \\
\hline $0.80-1$ & very high \\
\hline
\end{tabular}

The attributes are analyzed on the basis of their fuzzy values. Similarly for insulin dosage, different semantic descriptions have been set for the attributes as shown in table 2 .

Table 1. Semantic description of different levels of insulin dosage

\begin{tabular}{|c|c|}
\hline $\begin{array}{c}\text { Probability of Insulin } \\
\text { Dosage }\end{array}$ & Semantic description \\
\hline $0-0.3$ & low \\
\hline $0.3-0.6$ & med \\
\hline $0.6-1$ & high \\
\hline
\end{tabular}

II. Rule base: In the proposed work, two methodologies i.e. Type-1 FL and Interval Type-2 FL are used and there could be differences in their respective rule bases.

III. Comparison of the final output: The comparison is drawn on the basis of the difference of the outputs of Type1 FL and Interval Type-2 FL for both the diseases to understand three things:

i. The difference of the two methods by taking into account the predictions made by them.

ii. Analyzing whether the difference is uniform or nonuniform with the changing stages of the symptoms.

iii. The applicability of I-T2FL for medication prediction.

\subsection{Results}

1. For input $\mathrm{I}=[165,80,12,11,23,45,0.2]$, the output of the diabetes diagnosis prediction by Type-1 Fuzzy Logic is shown below.

fgt is high, sit is low, bmi is low, age is low and df is high. Type 1 FL Probability $=0.9159$. So, chances are very high. 
2. For input, $\mathrm{pgc}=140, \mathrm{si}=400, \mathrm{bmi}=23, \mathrm{mbp}=25$, age $=56, \mathrm{df}=0.55$. Type -2 fuzzy logic based diabetes diagnosis probability is given below.

$\mathrm{pgc}$ is high, si is high, bmi is low, mbp is medium, age is high. Type $1 \mathrm{FL}$ Probability $=0.5$ So, chances are medium. Type 2 FL Probability $=0.310$. So, chance are low.

3. For input, age $=56$, gender $=1$ (male), $\mathrm{cp}=3$, trestbps $=100$, thalach $=100$, exang $=0$, oldpeak $=0$, slope $=$ T2FL and T1FL is:

age is medium, cp is low, trestbps is low, thalach is low, exang is low, oldpeak is medium. Type $1 \mathrm{FL}$ probability $=0.9999999999999999$. So, the chances are very high. $\quad$ Type 2 FL Probability $=0.5681006902004474$. So, chances are medium.

4. For the input [fgt, bmi] $=[106,30.5]$, the output for insulin dosage according to type-1 fuzzy logic is:

fgt is low, bmi is med. Type-1 fuzzy dosage $=0.3201$, i.e., 'med' level of dosage

5. For the input [fgt, bmi $]=[106,30.5]$, the output for insulin dosage according to type-2 fuzzy logic is:

fgt is low, bmi is med. So, Type-2 fuzzy dosage = 0.2283 , i.e., 'low' level of dosage

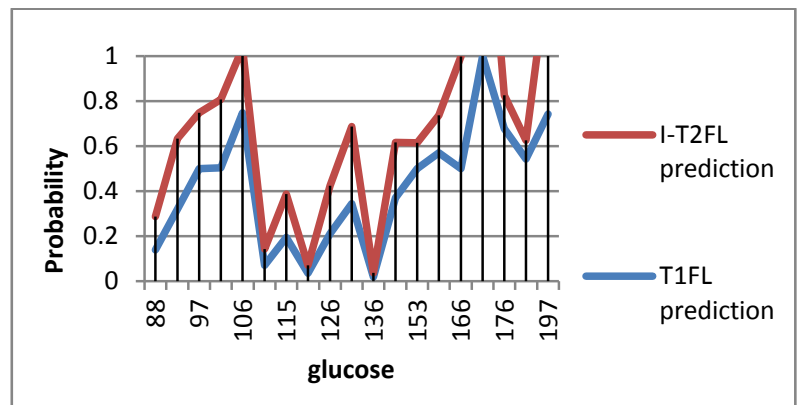

Figure 3: Graph depicting differences of the probabilities calculated by T1FL and I-T2FL

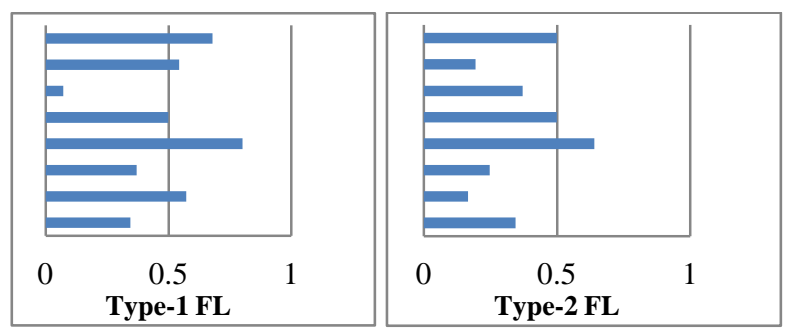

Figure 4: Predictions by Type-1 FL and Type-2 FL for positive diabetes diagnosis
I. The comparison of Type-1 FL and Interval Type-2 FL for type-2 diabetes diagnosis prediction: Figure 3 shows the difference of the outputs calculated for 20 inputs for diabetes.

a) There is no constant, but non-uniform gap between the Type-1 FL and Interval Type-2 FL based diagnosis prediction as the severity of the symptoms change.

b) The probability of diagnosis calculated by Interval Type-2 FL is always greater than that of the Type-1 FL.

From figure 4, it is noticeable that the predictions made by both approaches are nearly same in case of positive diagnosis of diabetes.

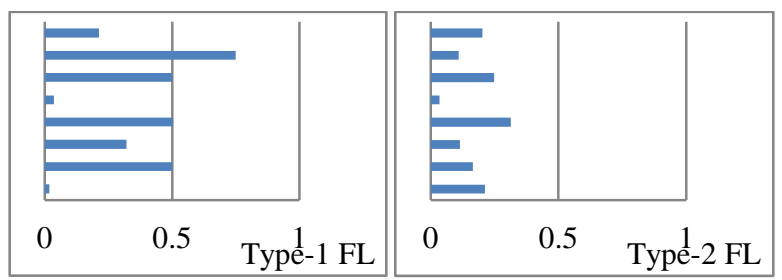

Figure 5: Predictions by Type-1 FL and Type-2 FL for negative diabetes diagnosis

However, in the case of negative diagnosis, the picture is quite different as shown in figure 5. Type-2 Fuzzy Logic attempts to achieve better accuracy (nearly 90\%), where Type-1 Fuzzy Logic achieves an accuracy up to just around $75 \%$.

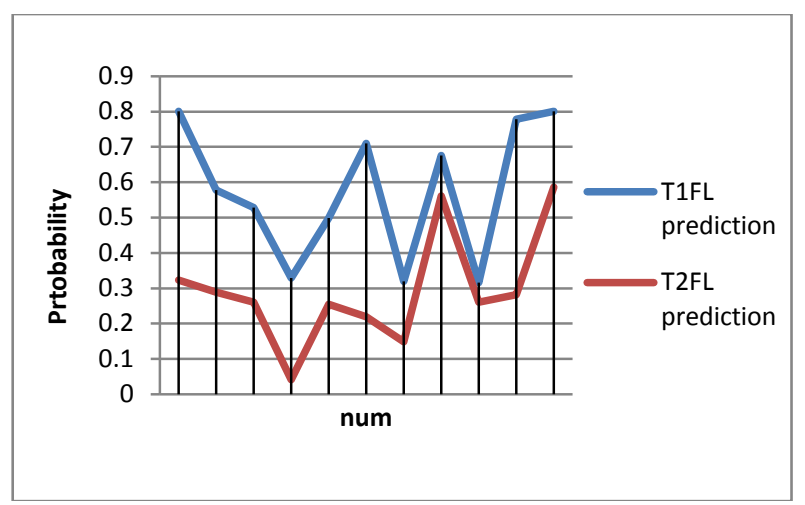

Figure 6: Graph depicting the probabilities calculated by T1FL and I-T2FL with respect to num

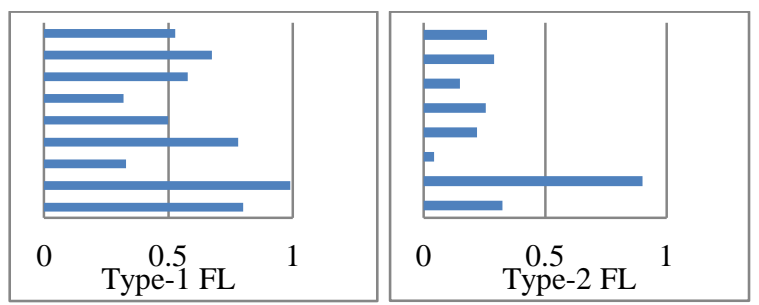

Figure 7: Probabilities calculated by Type-1 FL and Interval Type-2 FL for positive diagnosis of heart diseases

II. The comparison on the basis of heart related complication prediction: The figure 6 shows the gap between the predictions made by the two methodologies.

a) It is noticeable that unlike in the case of diabetes, Type-1 FL always predicts greater probability than Interval Type-2 FL. 
b) The gap between the two predictions is more uniform than that in the case of diabetes diagnosis prediction.

c) In case of heart diseases, Type-1 FL outperforms Interval Type-2 FL proving itself to be a better approach for diagnosis of heart related complications.

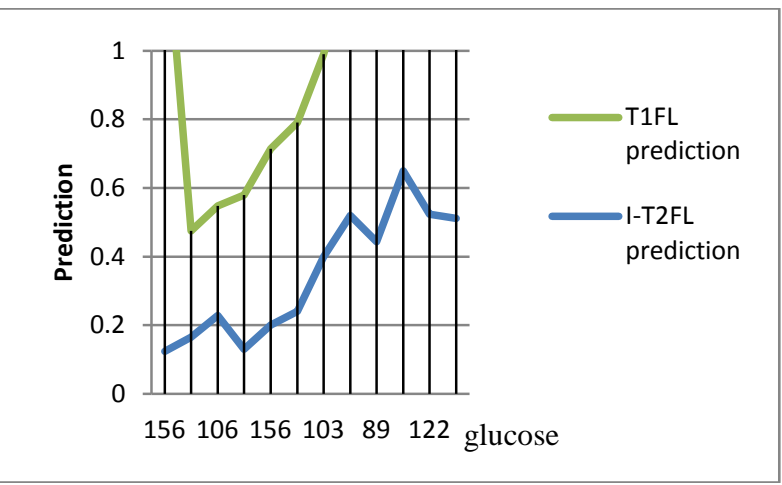

\section{Figure 8: Graphical comparison of insulin dosage} prediction

Figure 7 shows that T1FL is a better approach than the IT2FL for prediction of heart related diseases. T1FL is able to predict at an accuracy percentage of $99 \%$. Whereas, T2FL can only achieve accuracy upto $60 \%$.

Comparison of T1FL and I-T2FL in insulin dosage prediction: Figure 8 shows the expanding gap between the predictions of the two approaches. In the case of insulin. T1FL achieves an accuracy rate of $80 \%$, much higher than that of T2FL. It shows that T1FL performs better than T2FL where domain knowledge base is smaller in size.

\section{CONCLUSION AND FUTURE SCOPE}

The performance of Type-1 FL and interval Type-2 FL is different with respect to different diseases. It challenges the norm that the latter is always better than the former. The complexity of diabetes and heart related disease diagnosis is almost equal, yet the difference in the outputs of Type-1 FL and Type-2 FL triggers the need to further study these approaches in medical diagnosis.

For medication recommendation, the Interval Type- 2 FL is useful if the rule base is significantly larger and contains distinct rules with high confidence. And if the dataset is small like the diabetes medication data, Type-1 FL is a preferred approach.

The comparison of Type-1 FL and Interval Type-2 FL is presented only for diabetes and heart related complications which can also be performed on other diseases like HIV and TB. Due to non- availability of a universal dataset of these two diseases, the research in these diseases using fuzzy logic is quite limited. Besides this, various other metrics like complexity and time taken for diagnosis can be used for the comparison of the two approaches.

It is worth finding if Type-1 FL and Interval Type-2 FL can be used together in collaboration for better results and optimized use of resources. In medication, as both the approaches are unable to achieve a higher rate of accuracy, better approaches can be formulated by fusing these approaches with other soft computing methodologies.

\section{ACKNOWLEDGMENTS}

I would thank the Computer Science and Engineering Department of Thapar University for providing guidance and the facilities required for pursuing this research work. I would also thank my guide for providing the timely guidance and assistance.

\section{REFERENCES}

[1] Lee, C. and Wang, M. 2010. A Fuzzy System for Diabetes Decision Support Application. IEEE International Journal on Systems, Man, and Cybernetics. Vol. 41-No. 1, pp. 139-153.

[2] Calegari, S. and Sanchez, E. 2007. A Fuzzy OntologyApproach to improve Semantic Information Retrieval. Uncertainty Reasoning of Semantic Web. Vol. 327, pp. $1-6$.

[3] Abadi, D. N. M., Khooban, M. H., and Siahi, M. 2011. A Novel Automated Fuzzy Model for Diabetes Mellitus. In Proceedings of the IEEE 2nd International Conference on Control, Instrumentation and Automation (ICCIA), pp. 350-354.

[4] Gadaras, I. and Mikhailov, L. 2009. An interpretable fuzzy rule-based classification methodology for medical diagnosis. Artificial Intelligence in Medicine. Vol. 42No. 1, pp. 25-41.

[5] Yager, R. R. and Petry, F. E. 2006. A Multicriteria Approach to Data Summarization Using Concept Ontologies. IEEE Transactions on Fuzzy Systems. Vol. 14-No. 6, pp. 767-780

[6] Palma, J., Juarez, J. M., Campos, M., and Marin, R. 2006. Fuzzy theory approach for temporal model-based diagnosis: An application to medical domains. Artificial Intelligence in Medicine. Vol. 38-No. 2, pp. 197-218.

[7] Seising, R. 2006. From vagueness in medical thought to the foundations of fuzzy reasoning in medical diagnosis. Artificial Intelligence in Medicine. Vol. 38-No. 3, pp. 237-256.

[8] Straszecka, E. 2006. Combining uncertainty and imprecision in models of medical diagnosis. Information Sciences. Vol. 176-No. 20, pp. 3026-2059.

[9] Kalpana, M. and Kumar, A. V. S. 2011. Fuzzy Expert System for Diagnosis of Diabetes Using Fuzzy Determination Mechanism. International Journal of Computer Science and Emerging Technology. Vol. 2No. 6, pp. 39-45.

[10] Das, S. and Kar, S. 2014. Group decision making in medical system: An intuitionistic fuzzy softest. Applied Soft Computing. Vol. 24, pp. 196-211.

[11] Mahfouf, M., Abbod, M. F., and Linkens, D. A. 2001. A survey of fuzzy logic monitoring and control utilisation in medicine. Artificial Intelligence in Medicine. Vol. 21No. 1-3, pp. 27-41.

[12] Innocent, P. and John, R. 2004. Computer aided fuzzy medical diagnosis. Information Sciences. Vol. 162, pp. 81-104.

[13] Kahramanli, H. and Allahverdi, N. 2008. Design of a hybrid system for the diabetes and heart diseases. Expert Systems with Applications. Vol. 35-No. 1-3, pp. 82-89. 
[14] Gupta, N. K., Gupta, A., and Tyagi, P. K. 2014. Early Detection of Diabetes Patients using Soft Computing. In Proceedings of International Conference on Issues and Challenges in Intelligent Computing Techniques (ICICT). pp. 174-179.

[15] Anouncia, S. M., Madonna, L. J. C., Jeevitha, P., and Nandhini, R. T. 2013. Design of a Diabetic Diagnosis System Using Rough Sets. Cybernetics and Information Technologies. Vol. 13-No. 3, pp. 124-139.

[16] Lee, C. S., Wang, M. H., and Hagras, H. 2010. A Type2 Fuzzy Ontology and Its Application to Personal Diabetic-Diet Recommendation. IEEE Transactions on Fuzzy Systems. Vol. 18, pp. 374-395.

[17] Mendel, J. M., Hagras, H., and John, R. I. 2013. Guest Editorial for the Special Issue on Type-2 Fuzzy Sets and Systems. IEEE Transactions on Fuzzy Systems. Vol. 21No. 3, pp. 397-398.

[18] Yeh, C. W., Jeng, R., and Lee, S. 2011. An Enhanced Type-Reduction Algorithm for Type-2 Fuzzy Sets. IEEE Transactions on Fuzzy Systems. Vol. 19-No. 2, pp. 227240.
[19] Aladi, J. H., Wagner, C., and Garibaldi, J. M. 2014. Type-1 or INTERVAL Type-2 Fuzzy Logic Systems On the Relationship of the Amount of Uncertainty and FOU Size. In Proceedings of IEEE Conference on Fuzzy Systems. pp. 2360-2367.

[20] Campos-Delgado, D., Hernández-Ordoñez, M., Femat, R., and Gordillo-Moscoso, A. 2006. Fuzzy-Based Controller for Glucose regulation in Type-1 Diabetic Patients by Subcutaneous Route. IEEE Transactions on Biomedical Engineering. Vol. 51-No. 11, pp. 2201-2209.

[21] Grant, P. 2007. A new approach to diabetic control: Fuzzy logic and insulin pump technology. Medical Engineering \& Physics. Vol. 29-No. 7, pp. 824-827.

[22] Nazari, D., Abadi, M., Khooban, M. H., and Siahi, M. 2011. A Novel Automated Fuzzy Model for Diabetes Mellitus. In proceedings of IEEE 2nd International Conference on Control, Instrumentation and Automation (ICCIA). pp. 350-354

[23] Adeli, A. and Neshat, M. 2010. A Fuzzy Expert System for Heart Disease Diagnosis. In Proceedings of International MultiConference of Engineers and Computer Scientists. pp. 1-6. 